\title{
Synchrotron-based Transmission X-ray Microscopy (TXM) Observations of Fully Hydrated Blood Platelets and Their Activation Process
}

\author{
Nuri Yang, Hyun Woo Nho, Yogesh Kalegowda, Jin Bae Kim, Jaewoo Song, ${ }^{\dagger}$ Hyun-Joon Shin, ${ }^{\ddagger}$ and Tae Hyun Yoon ${ }^{*}$ \\ Laboratory of Nanoscale Characterization and Environmental Chemistry, Department of Chemistry, \\ College of Natural Sciences, Hanyang University, Seoul 133-791, Korea. ${ }^{*}$ E-mail: thyoon@gmail.com \\ 'Department of Laboratory Medicine, College of Medicine, Yonsei University, Seoul 120-752, Korea \\ "Pohang Accelerator Laboratory and Department of Physics, College of Physics, Pohang University, Pohang 790-784, Korea \\ Received December 16, 2013, Accepted January 29, 2014
}

\begin{abstract}
Platelets are anuclear discoid-shaped blood cells with key roles in human body. To understand the mechanisms of their activation process, it is required to have analytical imaging techniques capable of acquiring platelet images under fully hydrated conditions. Herein, for the first time, we demonstrate the capability of synchrotronbased transmission X-ray microscopy (TXM) to study platelets (resting and ADP activated) under hydrated and air-dried conditions. To confirm the biological imaging capability of TXM, fixed platelets were imaged and compared with whole mount electron microscopy (EM) images. TXM provided morphological information with sufficient spatial resolution with simple and quick sample preparation procedure. We also observed temporal changes during the platelet activation, which initially had a discoid shape $(0 \mathrm{~s})$, formed pseudopodia $(30 \mathrm{~s})$ and generated a network of fibrin $(5 \mathrm{~min})$. Our results clearly demonstrate the potential of TXM technique to study fully hydrated biological samples under in situ conditions.
\end{abstract}

Key Words : Transmission X-ray microscopy, Whole mount electron microscopy, Platelets, Platelet activation, In situ microscopy

\section{Introduction}

Platelets are anuclear discoid-shaped blood cells with a diameter of 1-3 $\mu \mathrm{m}$. They contain mitochondria, $\alpha$-granules, dense granules and other intra cellular organelles. ${ }^{1}$ As one of the smallest cells in our circulation system, blood platelets play an essential role in addressing blood vessel injury. Upon damage to a blood vessel, platelets rapidly gather at the location of damage, change their shape, and then secrete granule contents. This process involves platelet attachment to the exposed subendothelial tissue, platelet-platelet aggregation, platelet plug formation, and ultimately the arrest of bleeding. ${ }^{2-4}$ Secretory organelles, such as $\alpha$-granules and dense granules are known to be crucial in the secondary platelet response. ${ }^{5,6}$ There are 50-80 $\alpha$-granules ranging in size from 200 to $500 \mathrm{~nm}$ per platelet. $\alpha$-granules contain platelet-specific proteins such as heparin and coagulant factors, including fibrinogen., ${ }^{7,8}$ Other granule compounds include nucleotides (ATP, ADP, GTP and GDP) and cations, these are usually found in dense granules with a diameter of $150 \mathrm{~nm} .{ }^{8}$ Granule contents are all involved in hemostasis cascade reactions. Because the regulation of platelet secretion is fundamental to the functions of platelets, it is important to understand this regulation for future potential applications of platelets. More than 300 different molecules have been found in platelets, ${ }^{9}$ and $\alpha$-granules are considered to be the major storage and secretory organelle in platelets. ${ }^{4}$ However, the question of how such a diverse variety of proteins can be released in a regulated manner remains an open question. It was recently reported that various types of proteins are stored in separate $\alpha$-granules of platelets and released differentially upon stimulation. ${ }^{10}$ This claim is also supported by electron tomography data, which revealed morphological heterogeneity in the $\alpha$-granule population as well as spatial segregation of proteins within individual $\alpha$ granules. ${ }^{11}$ Therefore, it is important to understand the substructures of the platelet membrane system and their dynamics during the above processes.

Transmission electron microscopy (TEM) has contributed significantly to our current understanding of storage and release of granular contents from platelets and the ultrastructure of platelets. TEM can provide the spatial resolution necessary to determine platelet ultrastructure. Moreover, when combined with immunogold labeling, this electron microscopic technique provides information on the intracellular organization of platelet organelles and allows visualization of the intracellular distribution of proteins on a nanometer scale. However, a sophisticated sample preparation procedure involving dehydration, embedding, ultrathin sectioning, and several rounds of staining is required to prepare specimens for TEM, makes this technique a low throughput technique with the potential for distortion of the samples. Additionally, thin sections may not span entire organelles, therefore TEM observations of cross-sectioned specimens may only provide information about organelle fragments, rather than the entire organelle. ${ }^{12,13}$

To overcome these limitations of TEM, a novel microscopic approach to study storage, colocalization and secretion of biomolecules in human platelets has been developed: Transmission X-ray microscopy (TXM). TXM is a 

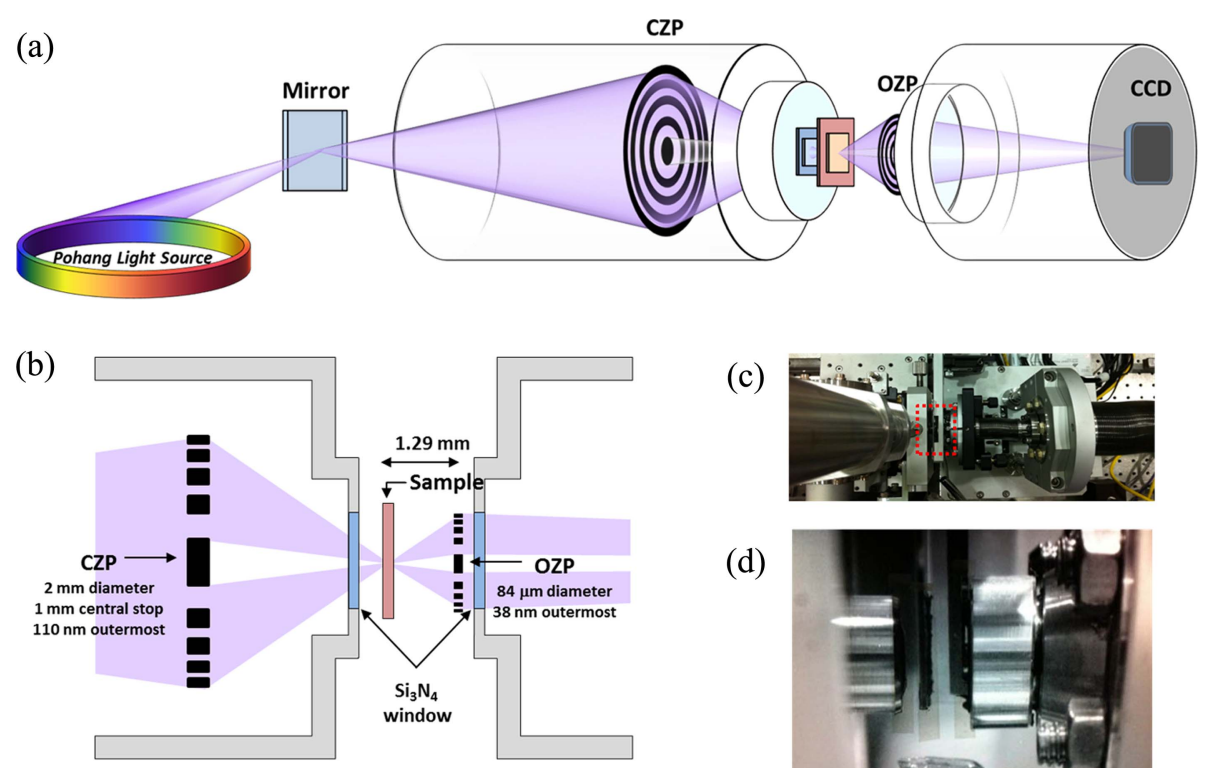

(c)

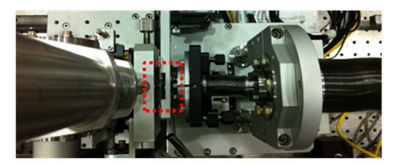

(d)

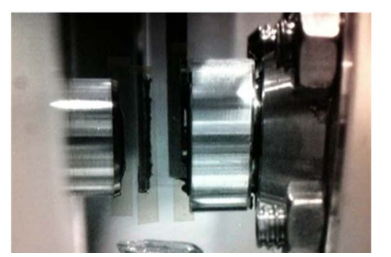

Figure 1. TXM instrumental setup in the Pohang Light Source, South Korea. (a) Schematic diagram of the TXM setup. (b) Zoomed view of the region between the condenser zone plate (CZP) and objective zone plate (OZP). (c) Top view of the sample holder. (d) Zoomed front view.

promising tool for nanometer-scale studies of biological samples because of the natural contrast mechanism of the water window (i.e., $284-540 \mathrm{eV}$ or $2.3-4.3 \mathrm{~nm}$ ). ${ }^{14-16}$

In this energy range, water is nearly an order of magnitude more transparent than organic matter, which provides the ideal contrast to observe biological specimens under wet conditions. The best spatial resolution of TXM is $12 \mathrm{~nm}$, which was achieved using the first order of diffraction of an objective zone plate (OZP) with an outermost zone width $(\Delta \mathrm{r})$ of $12 \mathrm{~nm} .{ }^{17}$ Such an achievement was made possible by recent technical advancements in $\mathrm{ZP}$ nanofabrication, such as double zone patterning and tilted zone patterning. However, since X-rays used in TXM studies also have enough energy to break chemical bonds and induce structural changes in biological tissues, ${ }^{18}$ it is important to pay special attention in minimizing radiation damage. ${ }^{19}$ For instance, cryo X-ray microscopy is one of the well-known methods often used to reduce radiation damage in samples composed of soft materials, such as polymeric materials and biological tissues. $^{20}$

In this work, we utilized a synchrotron-based TXM instrument with a spatial resolution of $40 \mathrm{~nm}$ to observe morphological changes of platelets upon activation by ADP. TXM provided subcellular level information on the spatial distribution of proteins within human platelets, which were minimally disturbed and maintained in a hydrated environment. We also discuss the complementary features of TXM and conventional TEM techniques.

\section{Experimental}

TXM Instrument. TXM images were collected at photon energies just below the oxygen edge, i.e. $500 \mathrm{eV}$ (corresponding to a wavelength of $\lambda=2.48 \mathrm{~nm}$ ) using the conv- entional transmission X-ray microscope endstation of the 10D beamline at Pohang Light Source (PLS, South Korea). Photon flux at the TXM setup is over $10^{10}$ photons/s $(0.1 \%$ $\mathrm{BW}) .^{21}$ The schematic layout of our TXM setup is presented in Figure 1. The setup includes a condenser zone plate (CZP, $2 \mathrm{~mm}$ diameter, $110 \mathrm{~nm}$ outermost zone width), an objective zone plate (OZP, $84 \mu \mathrm{m}$ diameter, $38 \mathrm{~nm}$ fine zone width, $1.29 \mathrm{~mm}$ first-order focal length), two $\mathrm{Si}_{3} \mathrm{~N}_{4}$ membrane windows (100 nm thickness and $500 \mu \mathrm{m}$ square, Norcada Co., Canada), a fully hydrated sample sandwiched between two $\mathrm{Si}_{3} \mathrm{~N}_{4}$ membranes, and a charge-coupled device (CCD, Peltier-cooled, in-vacuum back-illuminated 16-bit CCD $1024 \times 1024$ pixels, $13 \mu \mathrm{m}$ pixel size). The CZP was used to focus the synchrotron X-ray source on the sample, while the OZP was used to magnify and project the image of the sample onto the $\mathrm{CCD}$ camera. $\mathrm{Si}_{3} \mathrm{~N}_{4}$ membrane windows are used to allow the sample and the OZP to be positioned in air, while the other optical elements are in vacuum.

A fully hydrated platelet sample sandwiched between two $\mathrm{Si}_{3} \mathrm{~N}_{4}$ membranes was located between the CZP-side window and the OZP. Under normal operating conditions, the distance between the CZP-side window and the OZP is about 3-4 $\mathrm{mm}$. Due to this free space between the CZP-side window and the OZP, this TXM setup can be used for in situ computer-aided tomography studies of cellular objects or real-time imaging of chemical reactions in hydrous environments. Helium gas was blown slowly across the beam path to minimize X-ray absorption by air. The CCD camera was mounted 1.5-1.8 $\mathrm{m}$ downstream from the OZP.

Sample Preparation. Whole blood was obtained from normal healthy persons after informed consent and the study was approved by the Institutional Review Board (IRB) of Yonsei Severance hospital, Seoul. Whole blood was drawn into tubes containing ACD (Acid citrate dextrose : one sixth 
the volume of blood drawn). Platelet rich plasma (PRP) was obtained by centrifugation at 250 g-force for 15 minutes at $37^{\circ} \mathrm{C}$. Platelets were pelleted by centrifugation of PRP at $2200 \mathrm{~g}$-force for 15 minutes, followed by plasma removal. Platelet pellet was washed twice in Tyrode's buffer. PGI2 $(0.5 \mu \mathrm{M})$ was added to PRP and at every step of platelet resuspension to prevent premature activation of platelets. Finally, platelets were suspended in Tyrode's buffer containing $0.2 \mathrm{U} / \mathrm{mL}$ of apyrase, and the platelet count was adjusted to $300,000 / \mu \mathrm{L}$. Platelets were activated by ADP and fixed in $1 \%$ paraformaldehyde $0,0.5,5$, and 10 minutes after activation. Condensed and fixed platelets were sandwiched between two $\mathrm{Si}_{3} \mathrm{~N}_{4}$ membranes and observed using our TXM setup. PRP samples for whole mount electron microscopy (EM) analysis were prepared accordingly to the procedure described by Israels and colleagues. ${ }^{22}$ In brief, a single drop of PRP was transferred to a Formvar-coated copper grid. After incubation for $1 \mathrm{~min}$, excess PRP was removed by touching the grid edge with filter paper. A drop of $0.1 \%$ glutaraldehyde was placed on the grid for $1 \mathrm{~min}$ and removed in the same way. The grid was then washed with a drop of distilled water and allowed to air dry, after which platelet whole mount images were acquired.

\section{Results and Discussion}

To confirm the spatial resolution of the TXM setup we used in this study, different sized silica particles were observed. Figure 2 shows TXM images of $\mathrm{SiO}_{2}$ particles of $1 \mu \mathrm{m}, 500$ $\mathrm{nm}, 300 \mathrm{~nm}$, and $200 \mathrm{~nm}$ in diameter, respectively. These particles could be distinguished from one other. In particular, the $200 \mathrm{~nm} \mathrm{SiO}{ }_{2}$ nanoparticles, which are similar in size to the secretory granules contained in blood platelets, could be distinguished with good spatial resolution using the normal TXM setup we used in this study. Thus, it is expected that
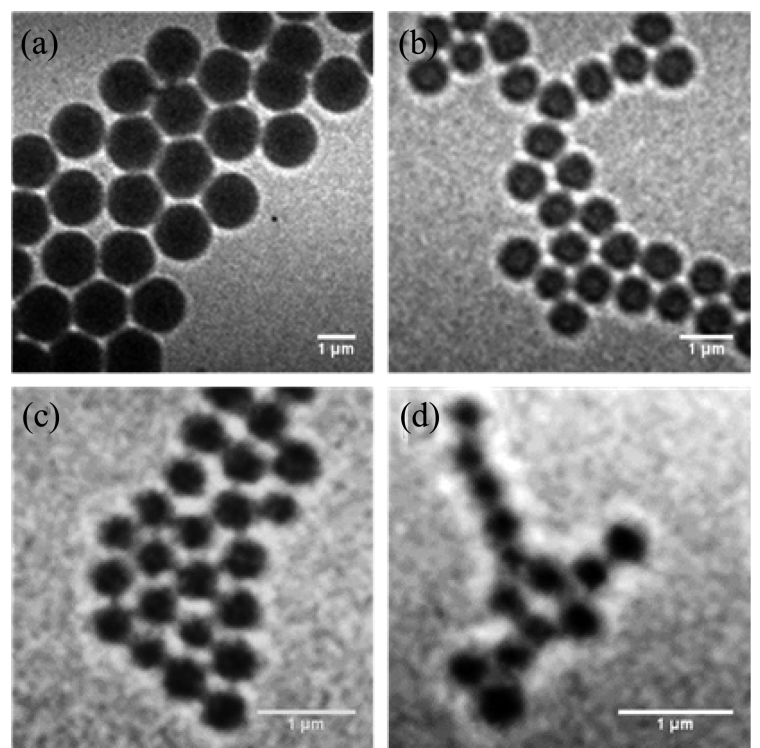

Figure 2. TXM images of $\mathrm{SiO}_{2}$ nanoparticles. (a) $1 \mu \mathrm{m}$, (b) 500 $\mathrm{nm}$, (d) $300 \mathrm{~nm}$ and (d) $200 \mathrm{~nm} \mathrm{SiO}$. Scale bar indicates $1 \mu \mathrm{m}$. we are able to identify granules within platelets using the current TXM experimental setup, as well as the shape of the intracellular granules.

Soft X-ray TXM generates 2-D images for 3-D thick biological samples, similar to light and electron microscopy techniques in transmission mode. ${ }^{23}$ To further confirm the biological imaging capability of TXM, fixed platelets were imaged by TXM and TEM techniques and we have compared the TEM images to those obtained using TXM to determine the limitations and advantages of TXM for studying the platelets.

Among the various TEM sample preparation techniques frequently used for blood platelet studies, we have chosen the technique of whole mount EM for unbiased comparison with the TXM technique. Figure 3(a) shows the TXM image of a fully hydrated whole platelet sandwiched between two $\mathrm{Si}_{3} \mathrm{~N}_{4}$ membranes, while Figure 3(b) presents the TEM image of a whole platelet mounted on a formvar-coated copper grid. In both images, platelets had a normal appearance with their sub-cellular components, such as secretory organelles (e.g., $\alpha$-granules and dense granules), mitochondria and open canalicular system (OCS). Dark regions of TXM platelet image are relatively dense organelles such as secretory granules and mitochondria, while the white regions indicate the OCS. The major storage components of platelets, such as $\alpha$-granules and dense granules, contain more organic compounds (e.g., proteins such as heparin and fibrinogen.) and absorb significantly more X-rays than the other part of the platelet with higher contents of water, resulting in significant contrast in this water window wavelength region. Therefore, in Figure 3(a), we have shown that it is possible to distinguish subcellular granules and OCS with the rest part of the platelet in the TXM image.

In the TEM image of whole mounted platelets shown in Figure 3(b), it showed better spatial resolution with clearer distinctions of sub-cellular components, such as granules and OCS. However, in the whole mount EM image shown in Figure 3(b), the presence of the filopodia cytoplasmic projections were not found, which was observed as fibrous structures in the TXM image shown in Figure 3(a). Additionally, in the whole mount EM image (Figure 3(b)), the platelet was abnormally expanded with a diameter of about $4.5 \mu \mathrm{m}$. Resting platelets were typically known to have a diameter of 2-3 mm with sub-cellular granules and organelles. ${ }^{1}$
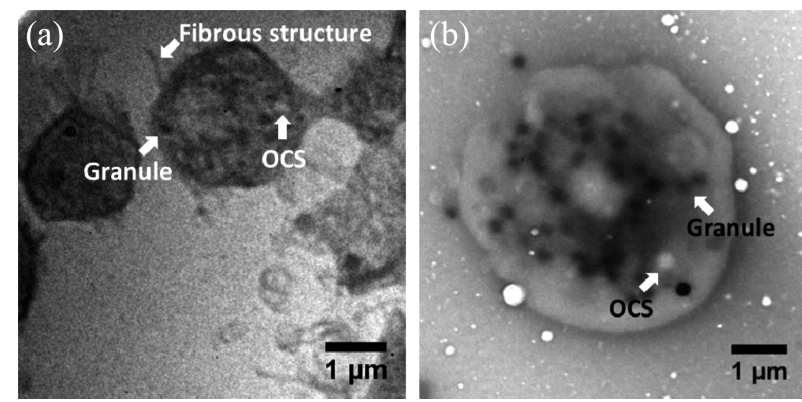

Figure 3. Non-activated resting platelets. (a) TXM and (b) whole mount EM images of a whole platelet sample. 

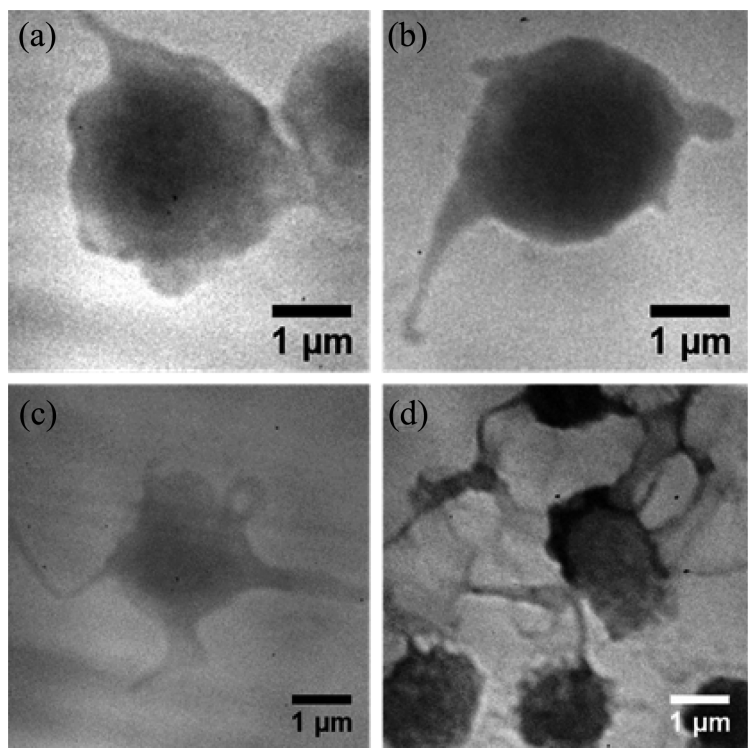

Figure 4. TXM images of platelet morphology changes as the progress of activation. Platelet activation was controlled by reaction time with ADP. (a) Resting platelet (reaction time $=0 \mathrm{~s}$ ), (b) platelet activated for $30 \mathrm{~s}$, (c) $5 \mathrm{~min}$, and (d) $10 \mathrm{~min}$ by ADP. Platelet samples were dropped between $\mathrm{Si}_{3} \mathrm{~N}_{4}$ membranes and airdried.

It is likely that the hydrated platelets were swollen when they were exposed to a vacuum condition during TEM measurement procedure due to the pressure difference between inside and outside of the cell. Those swollen platelets may then become flattened due to the leakage of intercellular water via vaporization. Additionally, it is also possible that the discrepancy in platelet sizes was an artifact related to extreme osmotic stress which platelets were subject to during the procedure for whole mount sample preparation.

Therefore, the comparison between TXM and TEM shown in Figure 3 confirmed us that the TXM has significant advantages over TEM particularly for biological samples. In contrast to the whole mount EM technique, which requires a high vacuum condition during observation, TXM can be performed for a fully hydrated sample under atmospheric pressure. In addition, because TXM samples are surrounded by water sandwiched between two $\mathrm{Si}_{3} \mathrm{~N}_{4}$ membranes, rather than being air- or vacuum- dried as is the case for TEM samples, they are expected to be more resistant to beam damage, since the radiation-induced radicals in aquatic environment can be rapidly quenched.

In Figures 4 and 5, platelet activation was induced by 10 $\mathrm{mM}$ of ADP and their temporal TXM images were presented. Platelet samples shown in Figure 4 were prepared by dropping PRP onto a $\mathrm{Si}_{3} \mathrm{~N}_{4}$ membrane and air-dried, while the TXM images shown in Figure 5 were acquired for the fully hydrated platelets by sandwiching PRP drop between two $\mathrm{Si}_{3} \mathrm{~N}_{4}$ membranes. As previously mentioned in the introduction section, platelet activation comprises morphological changes, release of storage substances and platelet aggregation. ${ }^{1}$ Morphological changes upon platelet activation are more clearly observed in Figure 4 rather than Figure 5. The
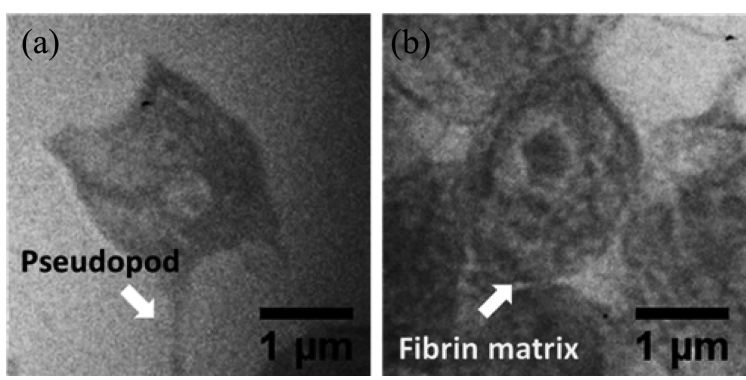

Figure 5. TXM images of a platelet activation series. (a) Activated platelet with a coagulant reaction time of $30 \mathrm{~s}$, (b) completely activated platelet with a coagulant reaction time of $5 \mathrm{~min}$. All samples were fully hydrated platelets via sandwiching PRP drop between two $\mathrm{Si}_{3} \mathrm{~N}_{4}$ membranes.

longer the activation time, the more formation of pseudopods were observed for the platelet. Upon plateletactivation, platelets were known to change their shape from discoid shape (resting state) to spherical (active state) and developed long pseudopods for efficient adhesion and aggregation. At the same time, the platelets were reported to secrete their granular contents to augment their own activation and to trigger activation of coagulation process. Platelet were also known to contains and secret several coagulation factors which are important in generation of hemostatic fibrin clot around platelet clumps. ${ }^{1}$

The activated platelet shown in Figure 5(a) displayed pseudopodia formation in response to the addition of ADP (reaction time: $30 \mathrm{~s}$ ). Actin and myosin in the cytoplasm were known to induce these morphological changes for clot retraction during bleeding. ${ }^{24}$ Following activation, those platelets activated for 5 min produced the network of fibrin as shown in Figure 5(b). This was expected, because the primary role of platelets is to arrest blood leakage, which was typically done by generating a mesh-like fibrin deposit that functions as a hemostatic plug. TXM enabled us to observe platelet activation and aggregation over time, which has never been possible previously. Furthermore, we could examine a sufficient numbers of platelets by TXM to take platelet-to-platelet variation into account.

\section{Conclusion}

This is the first synchrotron-based TXM imaging study of the fully hydrated blood platelets under resting and ADPactivated conditions. Comparisons of whole mount EM and TXM images revealed that TXM has a sufficient spatial resolution and contrast to observe morphological changes in sub-cellular levels. TXM provided morphological information about intact organelles and platelets with sufficient spatial resolution, while minimizing labor-extensive, time-consuming and artifact-causing sample preparation procedures of TEM observations. The ability to use TXM to observe platelets and their aggregates in a fully hydrated environment suggests that TXM can be a complementary technique to TEM, particularly for the study of platelet and their activation processes under in situ conditions. 
Acknowledgments. This research was supported by the Basic Science Research Program (NRF-2012R1A1A2009505) through the National Research Foundation of Korea (NRF) funded by the Korea government Ministry of Science, ICT and Future Planning (MSIP). In addition, this work was supported by MSIP and PAL, XFEL project, Korea. H Nho and HJ Shin also would like to acknowledge the financial support by the Basic Science Research Program (No. 20080062606, CELA-NCRC) through the NRF funded by the Korea government MSIP.

\section{References}

1. Kamath, S.; Blann, A. D.; Lip, G. Y. Eur. Heart J. 2001, 22, 15611571 .

2. Coller, B. S. J. Thromb. Haemost. 2011, 9, 374-395.

3. Jurk, K.; Kehrel, B. E. Internist (Berl) 2010, 51, 1086-1094.

4. Shi, G.; Morrell, C. N. Thromb. Res. 2011, 127, 387-390.

5. Lemons, P. P.; Chen, D.; Whiteheart, S. W. Biochem. Biophys. Res. Commun. 2000, 267, 875-880.

6. Shirakawa, R.; Higashi, T.; Tabuchi, A.; Yoshioka, A.; Nishioka, H.; Fukuda, M.; Kita, T.; Horiuchi, H. J. Biol. Chem. 2004, 279, 10730-10737.

7. Blair, P.; Flaumenhaft, R. Blood Rev. 2009, 23, 177-189.

8. Rendu, F.; Brohard-Bohn, B. Platelets 2001, 12, 261-273.

9. Whiteheart, S. W. Blood 2011, 118, 1190-1191.

10. Italiano, J. E., Jr.; Richardson, J. L.; Patel-Hett, S.; Battinelli, E.;
Zaslavsky, A.; Short, S.; Ryeom, S.; Folkman, J.; Klement, G. L. Blood 2008, 111, 1227-1233.

11. van Nispen tot Pannerden, H.; de Haas, F.; Geerts, W.; Posthuma, G.; van Dijk, S.; Heijnen, H. F. Blood 2010, 116, 1147-1156.

12. Meyer, D. A.; Oliver, J. A.; Albrecht, R. M. Microsc. Microanal. 2010, 16, 33-42.

13. Robinson, J. M.; Takizawa, T.; Vandré, D. D. J Histochem. Cytochem. 2000, 48, 487-492.

14. McDermott, G.; Le Gros, M. A.; Knoechel, C. G.; Uchida, M.; Larabell, C. A. Trends Cell Biol. 2009, 19, 587-595.

15. Magowan, C.; Brown, J. T.; Liang, J.; Heck, J.; Coppel, R. L.; Mohandas, N.; Meyer-Ilse, W. Proc. Natl. Acad Sci. USA 1997, 94, 6222-6227.

16. Meyer-Ilse, W.; Hamamoto, D.; Nair, A.; Lelièvre, S. A.; Denbeaux, G.; Johnson, L.; Pearson, A. L.; Yager, D.; Legros, M. A.; Larabell, C. A. J. Microsc. 2001, 201, 395-403.

17. Chao, W.; Kim, J.; Rekawa, S.; Fischer, P.; Anderson, E. H. Opt. Express 2009, 17, 17669-17677.

18. Wang, J.; Botton, G. A.; West, M. M.; Hitchcock, A. P. J. Phys. Chem. B 2009, 113, 1869-1876.

19. Yoon, T. H. Appl. Spectrosc. 2009, 44, 91-122.

20. Jacobsen, C.; Kirz, J. Nat. Struct. Biol. 1998, 5, 650-653.

21. Lim, J.; Shin, H. J.; Chae, K. H.; Hwang, C. C.; Hwang, H. N.; Hong, C. K. Rev. Sci. Instrum. 2010, 81, 063702.

22. Israels, S. J.; McNicol, A.; Robertson, C.; Gerrard, J. M. Br. J. Haematol. 1990, 75, 118-121.

23. Larabell, C. A.; Nugent, K. A. Curr. Opin. Struct. Biol. 2010, 20, 623-631.

24. George, J. N. Lancet 2000, 355, 1531-1539. 